\title{
Impulse Noise: A Real Threat for Workers and a Challenge for Acousticians
}

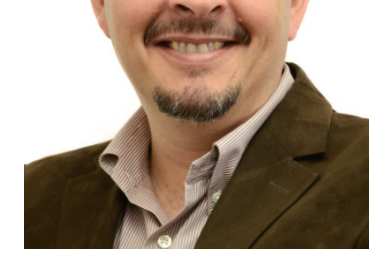

One of the most important occupational health problems in industrial environments is hearing loss due to impulsive noise. An impulsive noise is generated by the rapid release of compressed gases (impulse) or by the collision of solid objects (impact). It can be defined as the instantaneous change in sound pressure over a short period of time. Impulse durations can vary from tens of microseconds for small arms fire to several hundred milliseconds for a sonic boom or reverberating industrial impact.

It has been established that impulsive noises are more likely to cause noise-induced hearing loss than continuous noise of equal energy (Noise Health 2003; 5:63-73). The fundamental reason is that impulsive noises have a wide bandwidth and concentrate an enormous amount of sound energy in a small-time interval. The human ear's own protection mechanisms are also inefficient in activating the acoustic reflex. For this reason, it has been reported that even exposure above a peak of $140 \mathrm{~dB}$ (equivalent to a rms sound pressure of $200 \mathrm{~Pa}$ ) can cause permanent hearing loss and tinnitus, often when the impulse is unexpected (Audiol. NeuroOtol. 2002; 7:122-130). Exposure to high intensity impulses can also cause acoustic trauma and instantaneous mechanical damage to the inner ear (J. Acoust. Soc. Am. 1971; 49:1770-1777). Impulse noise exposure is common in metalworking industries, in construction, in shipyards, in mining, and among police and military personnel. In particular, the sound levels of impulsive noise, especially those generated by firearms or fireworks, can reach peak sound pressure level values in excess of 170-180 dB.

Definitions for impulse noise were introduced in the former American National Standards Institute (ANSI) standard S1.13 of 1971, and in the work of Pfander (Das knalltrauma, Springer, 1975) and Pfander et al. (J. Acoust. Soc. Am. 1980; 67: 628-633). The physical specification of an impulsive noise is further complicated when the impulses are mixed with a continuous noise which is very common in industrial environments. In fact, crest factors of up to $50 \mathrm{~dB}$ are commonly found in these environments.

The mechanism of hearing damage produced by impulsive noise is still under study. One difficulty in studying the effects of impulsive noise on hearing is that it is unethical to conduct research that exposes unprotected subjects to high-energy impulsive noise with the goal of determining permanent changes in hearing threshold. Because of this, most studies have been based on capturing temporal changes in hearing threshold after noise exposure. Other studies have been conducted on laboratory mammals (J. Acoust. Soc. Am. 1974; 56:1210-1221; Acustica 1985; 59:21-29). Although the most important parameters have been identified, the response to how much peak pressure, duration, frequency spectra, kurtosis and temporal spacing contribute to the mechanism of damage is even now unclear.
In the 1950s and 1960s, the U.S. National Academy of Sciences, through the Committee on Hearing, Bioacoustics and Biomechanics (CHABA), developed initial work on a risk-ofharm criterion for continuous noise and impulsive noise. The first proposal for an exposure limit for impulsive noise focused on gunfire and was based on a study by Coles et al. (J. Acoust, Soc. Am. 1968; 43:336-343). This proposal was not focused on industrial impulsive noise (impacts) and the noise dose was specified in terms of peak sound pressure and two aspects of the duration of a particular impulse, with correction factors for the number of impulses and for the angle of incidence of the sound wave with respect to the ear. It is in this criterion that the A- and B-durations were defined for the first time. Subsequently, two other impulse time metrics were defined for a pressure versus time plot: C-duration, used in Germany, and D-duration, used in the Netherlands.

Research on impulsive noise in the following years was scarce and was limited to a few studies in humans and animals. In that scenario, in 1971 the OSHA regulation was enacted in the USA, which established that exposure to impulse or impact noise should not exceed a peak SPL of $140 \mathrm{~dB}$. Thus, duration, spectral content, energy, and number of impulses were not considered in this recommendation. Clearly, gunshot noises could not be assessed according to this regulation as they always exceeded the recommended level.

Since the first CHABA proposal in 1968 had several limitations and questions due to the limited data available at that time, CHABA established a new working group in 1988 to revise this criterion. Fundamental to this work were data in animal models developed in the early 1990s. Price and Kalb (J. Acoust. Soc. Am. 1991; 90:219-227) developed a mathematical model to assess auditory risk in the presence of high-level impulses. The basic concept was to model the transfer function between free-field pressure and damage processes within the cochlea. The free-field waveforms served as input to the model that computed the transfer function of the head and the middle ear, and the subsequent motion of the ossicles (including nonlinear effects), to compute the basilar membrane displacement in the inner ear. This is the basis for what is now known as the Auditory Hazard Assessment Algorithm for Humans (AHAAH). The AHAAH is an electroacoustic model of the ear used to assess the risk of impulsive sounds to human hearing. The AHAAH model was initially developed based on the mechanical and fluid-dynamic properties of the ear. It involves an analysis of the wave motion of the basilar membrane based on the Wentzel-Kramers-Brillouin (WKB) method, which is commonly used in several fields of physics.

Conversely, Patterson and Hamernik (Noise-induced hearing loss, BC Decker, 1992) derived a spectral weighting function that showed that the energy contained in pulses at low frequencies should be reduced by up to $10 \mathrm{~dB}$ more than that corresponding to the A-weighting curve. 
In 1990 the ISO 1999 standard was published to consider impulsive noise of moderate value for application in industry. It was agreed to use the A-weighted equivalent level (LeqA) to assess impulsive noise levels up to $145 \mathrm{~dB}$ at the ear. There, it was established that exposure to a peak SPL higher than 140 $\mathrm{dB}$ will not be allowed. A similar criterion was adopted by the American Standards Institute (ANSI), in its ANSI S3.44 standard (1996), by the U.S. National Institute of Occupational Safety and Health (NIOSH) in 1998 and by the European Community in its directive $86 / 188$ of 1986 . One of the characteristics of the ISO 1999 standard is that it integrates the hazard of exposure to impulsive noise with exposure to continuous noise. Nevertheless, it has been established that this is not appropriate for levels exceeding $140 \mathrm{~dB}$ peak sound pressure level.

In the first report of the CHABA working group in 1992, based on the available scientific evidence, it was established that the application of the criterion should be for values greater than C-weighted $140 \mathrm{~dB}$ peak SPL. In the same report, it was recognized that the parameters of exposure to impulsive noise had to be based on the concepts of sound energy, spectral content, peak pressure level, duration, number of pulses, pulse mixture, and temporal spacing. This led to a modification of the exposure limits. It should be noted that, despite the time that has passed, impulsive noise considerations have not been incorporated by most countries into their noise legislation and regulations.

In a comparative study of occupational noise legislation in the Americas, Arenas and Suter (Noise Health 2014; 16:306319 ) showed that noise regulations in several nations treat impulsive noise separately from continuous noise. A common approach in some countries (generally those using an exchange rate $\mathrm{q}=5 \mathrm{~dB}$ ), has been to limit the number of impulses to a given maximum sound pressure during a working day, although the exact figures vary slightly. Alternatively, other nations have considered impulsive noise in conjunction with any continuous noise.

In 2003, NIOSH and the U.S. National Hearing Conservation Association (NHCA) developed a best practices workshop on impulsive noise, which was published in 2005 (Noise Control Eng. J. 2005; 53:53-60). This publication reported that research on risk criteria for hearing damage from impulsive noise has generated some specific criteria, some currently in use, including the 8-hour A-weighted equivalent sound level, denoted as LeqA8hrs (Scn. Aud. Suppl. 1998; 48:123-130).

For example, the U.S. Department of Defense has adopted an approach based on the AHAAH algorithm. The output of the AHAAH model is in "auditory risk units" (ARUs), which are linked to the damage resulting from basilar membrane displacements in the cochlea. A total of $500 \mathrm{ARU}$ is the maximum allowable dose for intermittent exposures, over a 24-hour period. Doses above 500 ARUs are expected to result in permanent hearing loss. For daily or near-daily occupational exposures, the limit is reduced to 200 ARU.

The three damage risk criteria contained in various versions of the standard MIL-STD-1474 differ significantly in their risk assessment. For example, the MIL-1474-E recommendation of 2015, employs ARU and the criterion of equal equivalent energy averaged over $100 \mathrm{~ms}$ intervals (LIAeq100ms), while the old MIL-1474-D recommendation of 1997, relied on the Aand $\mathrm{B}$-duration measurement, and provided a limiting category to impulsive noise.
Among the criteria for impulsive noise, mention can also be made of ANSI S12.7 (1986), which uses the sound exposure level (SEL), expressed as LxE, where $\mathrm{x}$ is the frequency weighting. Although less objective than the above, the use of the average peak level plot (measured as Lzpeak), expressed in pascals as a function of time in seconds, has also been recommended as a method of hearing damage assessment. Recently, kurtosis is being used as a descriptive metric because of the complexity of assessing the hearing risks of a complex noise environment. For this reason, in some circumstances it is difficult to establish when an exposure contains impulsive components that need to be assessed separately. Methods for estimating risk criteria for impulsive noise exposure damage remains a very active area of research.

Another difficulty in the analysis of impulsive noise in occupational environments is the metrological limitation. Impulsive noise peaks above $140 \mathrm{~dB}$ are very difficult to measure because many measuring instruments, which have been designed to measure continuous noise levels and intermittent sounds in the industrial environment, are not capable of accurately acquiring such intense sounds. In addition, due to the short duration of an impulsive noise, a very high data acquisition rate of more than 100,000 samples per second is required. However, standard dosemeters and sound level meters do not exceed 50,000 samples per second (generally $48 \mathrm{kHz}$ ). Therefore, these instrumental limitations can seriously underestimate peak levels or other pulse parameters. More recently, some manufacturers have developed specialized sound pressure level instruments equipped with high-dynamic range microphones that can measure pulses up to $160 \mathrm{~dB}$ at an exceptionally high sampling rates. However, this type of instrumentation is still very expensive which makes its use restricted to scientific research.

Regarding the assessment of the effectiveness of hearing protection devices (HPDs) to impulsive noise, the literature is scarce. The efficiency of PAs is, in general, assessed by the procedure of ISO 4869-2 of 2018. This standard defines three methods for continuous noise measurements (octave bands, HML and SNR). This same standard states that these methods may not be suitable for use with peak level measurements. Currently, the main guidance document for assessing the attenuation of a HPD is Annex B of the European Standard EN458 of 2016. This defines three types of impulsive noise corresponding to typical impulsive sources. The described method does not use any additional metrics to the $\mathrm{C}$-weighted peak level and is based on HML values obtained by means of ISO 4869-2. Obviously, the process is highly subjective and is considered insufficient to adequately assess the efficiency of a hearing protector in the presence of impulsive noise.

There are numerous challenges in the study of impulsive noise, its hearing risk assessment criteria, how to measure it and how to select the correct hearing protectors for this type of noise. Thus, it is expected that research will continue to solve the existing scientific and technological challenges in this field.

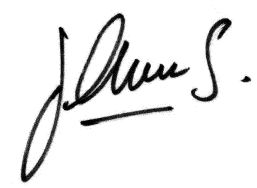
Jorge P. Arenas
Fellow IIAV
Editor-in-Chief IJAV 